\title{
XMM-Newton observation of the NLS1 galaxy Ark 564
}

\section{Spectral analysis of the time-average spectrum}

\author{
I. E. Papadakis ${ }^{1}$, W. Brinkmann ${ }^{2}$, M. J. Page ${ }^{3}$, I. Mc Hardy ${ }^{4}$, and P. Uttley ${ }^{5}$ \\ 1 Physics Department, University of Crete, PO Box 2208, 71003 Heraklion, Crete, Greece \\ e-mail: jhep@physics.uoc.gr \\ 2 Max-Planck-Institut für extraterrestrische Physik, Giessenbachstrasse, 85740 Garching, Germany \\ 3 MSSL, University College London, Holmbury St. Mary, Dorking, Surrey RH5 6NT, UK \\ 4 School of Physics and Astronomy, University of Southampton, Southampton SO17 1BJ, UK \\ 5 Sterrenkundig Instituut, Universiteit van Amsterdam, Kruislaan 403, 1098 SJ, Amsterdam, The Netherlands
}

Received 1 May 2006 / Accepted 29 September 2006

\section{ABSTRACT}

\begin{abstract}
Context. We present the results from the spectral analysis of the time-average spectrum of the Narrow Line Seyfert 1 (NLS1) galaxy Ark 564 from a $\sim 100$ ks XMM-Newton observation.

Aims. Our aim is to characterize accurately the shape of the time-average, X-ray continuum spectrum of the source and search for any emission and/or absorption features in it.

Methods. We use the XMM-Newton data to obtain the X-ray spectrum of the source and we fit various spectral models to it.

Results. The time-average, $3-11 \mathrm{keV}$ spectrum is well fitted by a power-law of slope 2.43 . We detect a weak (equivalent width $\sim 80 \mathrm{eV}$ ) emission line at $\sim 6.7 \mathrm{keV}$, which implies emission from ionized iron. There is no compelling evidence for significant broadening of the line.We also detect a possible Doppler shifted absorption line at $8.1 \mathrm{keV}$. At energies $\lesssim 2 \mathrm{keV}$, the spectrum is dominated by a smooth soft excess component which can be fitted well either by a model consisting of two black body components $(k T \sim 0.15$ and $0.07 \mathrm{keV}$ ) or by a model consisting of a black body plus reflection from a relativistically-blurred, photoionized disc. We detect a broad, shallow flux deficit in the $0.65-0.85 \mathrm{keV}$ band, reminiscent of the iron unresolved transition array (UTA) features. We detect neither a strong absorption edge around $0.7 \mathrm{keV}$ nor an emission line around $1 \mathrm{keV}$.

Conclusions. The soft excess emission is consistent with being reflected emission from a geometrically flat disc, with solar abundances, illuminated by an isotropic source. The weakness of the iron line emission can be explained by relativistic blurring. The UTA feature implies the presence of warm material with a column density of $2-5 \times 10^{20} \mathrm{~cm}^{-2}$. If the absorption line at $8.1 \mathrm{keV}$ corresponds to FeXXVI K $\alpha$, it suggests the presence of highly ionized absorbing material with $N_{\mathrm{H}}>10^{23} \mathrm{~cm}^{-2}$, outflowing at a high velocity of $\sim 0.17 \mathrm{c}$.
\end{abstract}

Key words. galaxies: active - galaxies: Seyfert - galaxies: individual: Ark 564 - X-rays: galaxies

\section{Introduction}

Narrow-line Seyfert 1 (NLS1) galaxies are a peculiar group of active galactic nuclei (AGN) characterized by their distinct optical emission line properties (Osterbrock \& Pogge 1985). In hard X-ray studies NLS1 galaxies comprise less than $10 \%$ of the Seyfert galaxies. However, from the ROSAT All-Sky Survey it became clear that about half of the AGN in soft X-ray selected samples are NLS1 galaxies (Grupe 1996; Hasinger 1997). Boller et al. (1996) and Wang et al. (1996) found from ROSAT observations that the soft X-ray spectra of NLS1 galaxies are systematically steeper than those of broad line Seyfert 1 galaxies. The popular explanation of the differences in X-ray properties between narrow and broad line Seyferts is that the former objects have relatively low black hole masses and high accretion rates (e.g. Pounds et al. 1995; Puchnarewicz et al. 2001).

Ark 564 is the X-ray brightest NLS1 galaxy with a $2-10 \mathrm{keV}$ flux of $\sim 2 \times 10^{-11} \mathrm{erg} \mathrm{cm}^{-2} \mathrm{~s}^{-1}$ (Turner et al. 2001) and shows large amplitude variations on short time scales (Leighly 1999a). Ark 564 was observed for a period of $\sim 35$ days in June/July 2000 by ASCA as part of a multi-wavelength AGN Watch monitoring campaign (Turner et al. 2001). The timing behavior of the source was studied by Edelson et al. (2002), who found no evidence of lags between any of the energy bands studied and that the fractional variability amplitude was almost independent of energy. Using the same ASCA data, Papadakis et al. (2002) reported a " -1 to -2 " slope break in the power spectrum at high frequencies $\left(\sim 2 \times 10^{-3} \mathrm{~Hz}\right)$. On the other hand, Pounds et al. (2001) detected a "zero to -1 " low frequency PSD slope break at $\sim 1 / 13$ days $^{-1}$, using long term RXTE monitoring observations. When combined, these two results support the idea of a small black hole mass, and hence high accretion rate, in Ark 564. Finally, Gliozzi et al. (2002) found no statistically significant indications of non-stationarity in the ASCA light curves. Furthermore, using nonlinear techniques they were able to demonstrate that the source behaves differently in the high and low flux states.

From the ASCA long-look data the $2-10 \mathrm{keV} \mathrm{X}$-ray spectrum was found to be quite steep $(\Gamma=2.45-2.72)$. It also showed a strong Fe $\mathrm{K} \alpha$ line with an equivalent width of $E W \sim$ 350-650 eV, depending on the fitted model, which seemed to originate in highly ionized gas (Turner et al. 2001). The presence of a soft excess was established but its exact form could not be constrained. From a 50 ks observation of Ark 564 with the Chandra HETGS Matsumoto et al. (2004) confirmed the steep 
power-law $(\Gamma=2.54 \pm 0.06)$ above $2 \mathrm{keV}$ and claimed the detection of an edge-like absorption feature at $0.712 \mathrm{keV}$. An emission like feature at $\sim 1 \mathrm{keV}$ has been reported from various lowresolution spectra from ROSAT, ASCA and BeppoSax (Brandt et al. 1994; Turner et al. 1999; Comastri et al. 2001), but its origin remains unclear. Comastri et al. (2001) claimed the detection of a narrow iron emission line at $\sim 6.8 \mathrm{keV}$ and an absorption edge at $\sim 9.5 \mathrm{keV}$ in the source rest frame from BeppoSax observations. Vaughan et al. (1999) detected an edge at $8.5 \mathrm{keV}$ in combined $A S C A$ and $R X T E$ data, which they attributed to reflection from a strongly irradiated disc. Finally, Vignali et al. (2004) presented results from the analysis of two short (12 and $\sim 6 \mathrm{ks})$ XMM-Newton observations. They also found a steep hard power-law $(\Gamma \sim 2.52-2.56)$. The soft excess was fitted with a black body component with a temperature of $\sim 140 \mathrm{eV}$ plus an absorption edge at $\sim 740 \mathrm{eV}$, with an optical depth, $\tau$, of $\sim 0.4$. For both pointings the quality of the best fits was only moderate $\left(\chi_{\text {red }}^{2} \sim 1.2\right)$ despite the limited photon statistics.

In this paper we present the results from the analysis of the time-average spectrum of the source resulting from a $100 \mathrm{ks}$ XMM-Newton observation of Ark 564. After describing the observation details, in Sect. 3 we present a short analysis of the temporal behaviour of the source. In Sects. 4 and 5 we describe in detail the spectral analysis of the data and we discuss the X-ray spectral properties of Ark 564, respectively. Our conclusions are presented in Sect. 6.

\section{Observation and data analysis}

Ark 564 was observed with XMM-Newton from 2005 January 5, 19:47 to 2005 January $6,23: 16$ for 101774 s (obsID: 0206400101). The PN and the two MOS cameras were operated in Small Window mode with a medium filter. The EPIC data were reprocessed with the XMMSAS version 6.5 and for the spectral analysis we used the most recent versions of the response matrices.

The background count rate was very low (in total less than $0.6 \%$ of the source count rate), apart from two small, short flares at the beginning of the observation. Data from these periods were disregarded from the spectral analysis.

With an average count rate of $\sim 30 \mathrm{cts}^{-1}$ photon pileup is negligible for the PN detector, as was verified using the XMMSAS task epatplot. Source counts were accumulated from $27 \times 26$ RAW pixels ( 1 RAW pixel $\sim 4.1^{\prime \prime}$ ) around the position of the source. Background data were extracted from a similar, source free, region on the chip. In order to minimize the effects of any calibration uncertainties, we selected only single events for the spectral analysis (PATTERN $=0$ and FLAG $=0$; for details of the instruments see Ehle et al. 2005) in the energy range from $300 \mathrm{eV}$ to $12 \mathrm{keV}$. In total $\sim 2 \times 10^{6}$ photons were accumulated in an integration time of $\sim 69300 \mathrm{~s}$.

For the MOS data, with average count rates of $\sim 8 \mathrm{cts} \mathrm{s}^{-1}$, pile-up is not negligible. We therefore accumulated the source counts from a ring of outer radius $45^{\prime \prime}$ excluding the innermost 12 . 5 centered on the position of Ark 564. The background data were extracted from a similar region from another chip. Events with PATTERN $\leq 12$ and FLAG $=0$ were used for the analysis. In a net exposure time of $\sim 96 \mathrm{ks}, \sim 2.3 \times 10^{5}$ photons were selected for each of the two MOS cameras in the $0.3-10 \mathrm{keV}$ energy band.

Finally, the RGS data were reduced using RGSPROC in XMMSAS 6.5 and the latest available calibration files (November 2005). Residuals in the current effective area calibration were corrected using the data/model ratio of a power-law fit

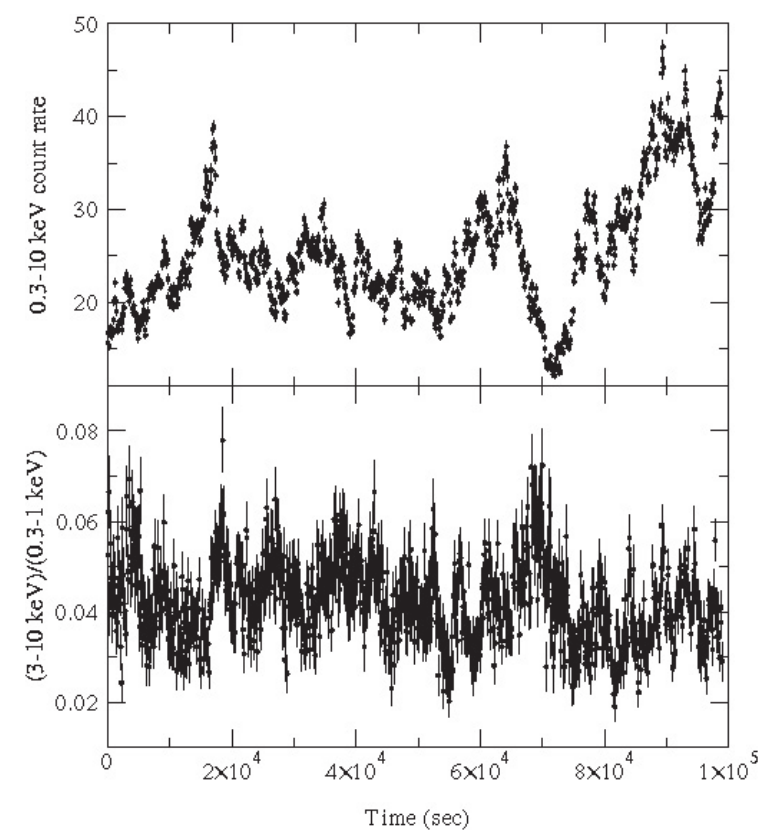

Fig. 1. Upper panel: background subtracted PN light curve of Ark 564 in the $0.3-10 \mathrm{keV}$ energy band. Note that in reality the average count rate is slightly larger, as we have not corrected the count rate for the $71 \%$ live time of the Small Window mode of the detector. Lower panel: the corresponding $(3-10 \mathrm{keV}) /(0.3-1 \mathrm{keV})$ hardness ratio curve. The time binning in both panels is $100 \mathrm{~s}$.

to the rev 0084 observation of the continuum source Mrk 421. The first and second order spectra and response matrices from RGS1 and RGS2 were resampled to the first order RGS1 spectrum, then combined to produce a single spectrum and a single response matrix.

\section{Timing analysis}

Ark 564 is a highly variable source. A detailed timing analysis of the observed light curves in various energy bands will be presented in McHardy et al. (in preparation). Recently, Arevalo et al. (2006) combined the data from the $100 \mathrm{ks} X M M-N e w t o n$ observation with those from the month-long monitoring $A S C A$ observation to calculate the time lags and coherence functions between various energy bands.

The upper panel of Fig. 1 shows the PN $0.3-10 \mathrm{keV}$ background subtracted light curve, binned in $100 \mathrm{~s}$ intervals. The source is highly variable on all sampled time scales. The max/min variability amplitude is of the order of $\sim 4$, while the fractional variability amplitude (corrected for the experimental noise) is $f_{\text {rms }}=24.8 \pm 0.1 \%$ (the error accounts only for the measurement error in the light curve points, and has been estimated according to the prescription of Vaughan et al. (2003)). We also considered light curves in 3-10 keV and $0.3-1 \mathrm{keV}$ as representative of the "hard" and "soft" energy bands, respectively. The hard band variations are of larger amplitude $\left(f_{\text {rms, hard }}=27.2 \pm\right.$ $0.4 \%$ as opposed to $f_{\text {rms,soft }}=24.7 \pm 0.1 \%$ ). This difference suggests the presence of spectral variations.

In the lower panel of Fig. 1 we plot the (3-10 keV)/ (0.3-1 keV) hardness ratio as a function of time. This plot reveals that the observed flux variations are indeed associated with spectral variations as well. Although significant, they are of rather small amplitude. In some cases, like for example around $\sim 18$ and $75 \mathrm{ks}$ from the start of the observation, the spectrum 


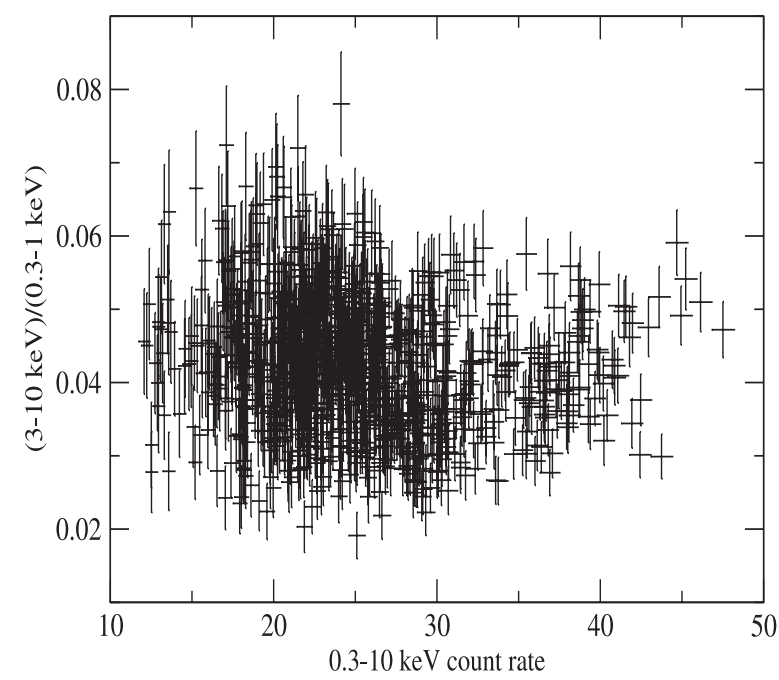

Fig. 2. The $(3-10 \mathrm{keV}) /(0.3-1 \mathrm{keV})$ hardness ratio plotted as a function of the total count rate.

becomes clearly harder as the flux decreases. However, it is not obvious that this trend holds at all times. In fact, the "hardness ratio versus count rate" plot (Fig. 2) demonstrates that the relation between flux and spectral variations is far from clear and simple. The results from a detailed analysis of the observed spectral variations will be presented in a subsequent paper (Brinkmann et al., in preparation).

Using the results from the spectral analysis presented below, the average $0.3-10 \mathrm{keV}$ flux amounts to $\sim 1 \times 10^{-10} \mathrm{erg} \mathrm{cm}^{-2} \mathrm{~s}^{-1}$ which results in an X-ray luminosity of $L_{0.3-10 \mathrm{keV}}=6.5 \times$ $10^{43} \mathrm{erg} \mathrm{s}^{-1}$, assuming a redshift of $z=0.0247$ and a Friedman cosmology with $H_{0}=75 \mathrm{~km} \mathrm{~s}^{-1} \mathrm{Mpc}^{-1}, q_{0}=0.5$. A substantial part of the flux is emitted in the soft energy band. The average $2-10 \mathrm{keV}$ flux amounts to $1.5 \times 10^{-11} \mathrm{erg} \mathrm{cm}^{-2} \mathrm{~s}^{-1}$ which is comparable to the mean flux of $2 \times 10^{-11} \mathrm{erg} \mathrm{cm}^{-2} \mathrm{~s}^{-1}$ during the 35 day long ASCA observation in June 2000 (Turner et al. 2001).

\section{Spectral analysis of the PN and MOS data}

For the spectral analysis the source counts were grouped with a minimum of 30 counts per energy bin. Spectral fits have been performed with the XSPEC v11.3 package. Spectral responses and the effective area for the PN and MOS spectra were generated with the SAS commands rmfgen and arfgen. The errors on the best-fitting model parameters represent the $90 \%$ confidence limits for one interesting parameter, and in the cases we report upper limits, these correspond to the $99 \%$ confidence limit. The energy of the emission or absorption features are given in the rest frame of the source. Finally, we consider a model as providing an acceptable fit to the data if the goodness of fit is better than the $5 \%$ confidence level, and we accept that the addition of a model component is necessary if the quality of the model fitting is improved at more than the $95 \%$ significance level.

\subsection{The hard band EPIC PN spectrum}

The spectral complexity of Ark 564 in the $0.3-10 \mathrm{keV}$ band is well known from previous observations. We therefore started by fitting the hard band, i.e. 3-11 keV, PN spectrum with a simple power-law. The Galactic absorption was modeled using PHABS in XSPEC and the abundance table of Lodders (2003), keeping

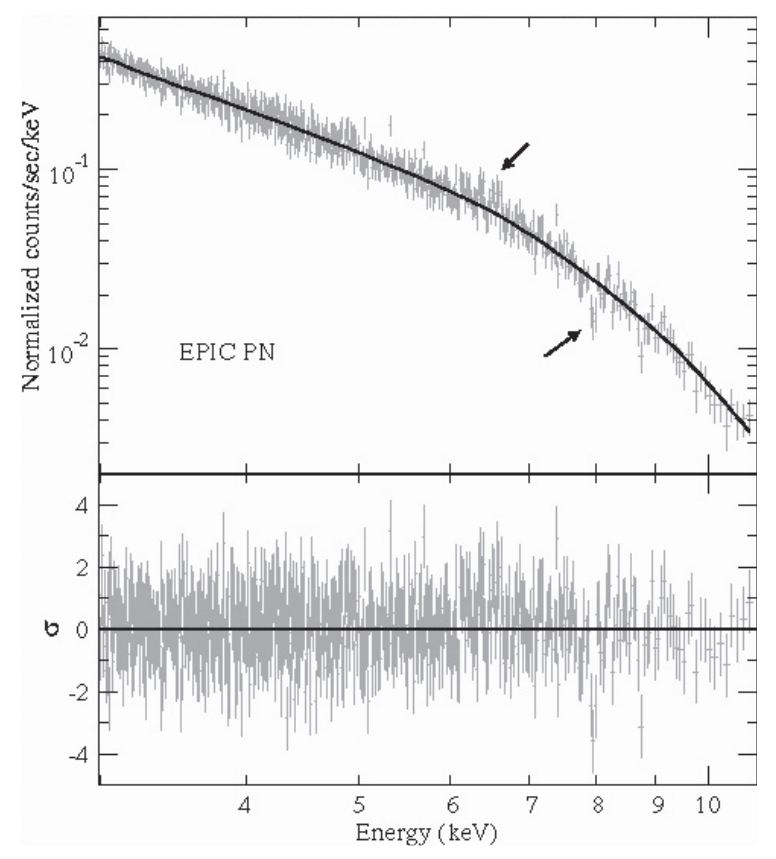

Fig. 3. Plot of the power-law fit (upper panel) and residuals (lower panel) to the PN data in the 3-11 keV energy band. Notice the residuals in the $\mathrm{Fe}$ line region and the absorption feature around $\sim 8 \mathrm{keV}$ (indicated with the arrows in the upper panel). In this, and all subsequent similar plots, the best model fitting residuals are plotted in terms of $\sigma$ with error bars of size one.

the value of the interstellar absorption fixed at $N_{\mathrm{H}}=6.4 \times$ $10^{20} \mathrm{~cm}^{-2}$ (Dickey \& Lockman 1990).

The fit is acceptable with a reduced $\chi_{\text {red }}^{2} /$ d.o.f. $=0.987 / 631$. The best fitting results are listed in Table 1 (model "PL"). Figure 3 shows the best PL model fit to the PN data and the corresponding residuals. The only apparent systematic deviations (indicated with arrows in Fig. 3) appear at $\sim 6-6.5 \mathrm{keV}$ (where iron line emission features are expected) and around $8 \mathrm{keV}$, where a narrow absorption feature appears. Even if real, it is obvious that these features are rather weak.

We repeated the fit with the addition of a Gaussian emission line. Initially we kept the width of the line fixed at $\sigma=100 \mathrm{eV}$ (i.e. smaller than the PN's resolution at these energies). The results are listed in Table 1 (model "PL+NGL"). The addition of the narrow line improves the goodness of fit by $\Delta \chi^{2}=13$ for 2 d.o.f., significant at the $99.9 \%$ level. The line's energy of $6.67_{-0.10}^{+0.08} \mathrm{keV}$ is indicative of emission from Fe XXV. Inspection of the residuals suggests a possible second emission line feature at $\sim 6.4 \mathrm{keV}$. Consequently, we added a second Gaussian line, keeping the energy of the first line fixed at its best fitting value of $6.67 \mathrm{keV}$. We list the results in Table 1 (model "PL+2 NGLs"). The quality of the fit improves only by $\Delta \chi^{2}=3.4$ for 1 d.o.f., which is not significant. We then examined whether the fit would improve if we let the width of the $6.7 \mathrm{keV}$ emission line vary as a free parameter. This improves the fit by $\Delta \chi^{2}=2$ for 1 d.o.f., which again is not significant. The best fitting $\sigma$ value of $\sim 0.2 \mathrm{keV}$ (Table 1 , model "PL+BGL") is only slightly larger than the PN's intrinsic resolution of $\sim 150-170 \mathrm{eV}$ at $6-7 \mathrm{keV}$.

We then repeated the model fitting with a DISKLINE model (Fabian et al. 1989) substituted for the Gaussian emission line. Since the emission feature in the PN spectrum is not strong, we kept the inner and outer disc radii fixed to 10 and 1000 gravitational radii $\left(r_{\mathrm{g}}\right)$, respectively, the emissivity index to -2 , and the inclination angle to 30 degrees. The best fitting results are listed 
Table 1. Results from spectral fitting in the $3-11 \mathrm{keV}$ energy band assuming fixed Galactic $N_{\mathrm{H}}=6.4 \times 10^{20} \mathrm{~cm}^{-2}$. The letter $f$ indicates parameters whose value was kept fixed during the model fitting.

\begin{tabular}{|c|c|c|c|c|c|c|c|c|c|}
\hline & Model & $\Gamma$ & $E_{\text {line }}(\mathrm{keV})$ & $\sigma(\mathrm{keV})$ & $E W(\mathrm{eV})$ & $E_{\text {line }, 2}(\mathrm{keV})$ & $\sigma_{2}(\mathrm{keV})$ & $E W_{2}(\mathrm{eV})$ & $\chi_{\text {red }}^{2} /$ d.o.f. \\
\hline $\mathrm{PN}$ & $\mathrm{PL}$ & $2.44 \pm 0.03$ & - & - & - & - & - & - & $0.987 / 631$ \\
\hline PN & $\mathrm{PL}+\mathrm{NGL}$ & $2.46 \pm 0.03$ & $6.67_{-0.10}^{+0.08}$ & $f(0.1)$ & $44 \pm 20$ & - & - & - & $0.97 / 629$ \\
\hline $\mathrm{PN}$ & $\mathrm{PL}+2$ NGLs & $2.47 \pm 0.03$ & $f(6.67)$ & $f(0.1)$ & $40 \pm 20$ & $6.35_{-0.16}^{+0.13}$ & $f(0.1)$ & $23_{-19}^{+17}$ & $0.966 / 628$ \\
\hline PN & $\mathrm{PL}+\mathrm{BGL}$ & $2.47 \pm 0.03$ & $6.60_{-0.15}^{+0.14}$ & $0.22_{-0.17}^{+0.38}$ & $66_{-32}^{+60}$ & -0.10 & - & - & $0.968 / 628$ \\
\hline PN & $\mathrm{PL}+\mathrm{DL}$ & $2.47 \pm 0.03$ & $6.59_{-0.09}^{+0.08}$ & - & $76 \pm 30$ & - & - & - & $0.965 / 629$ \\
\hline PN & $\mathrm{PL}+\mathrm{DL}+\mathrm{ABL}$ & $2.45 \pm 0.03$ & $6.6 \pm 0.1$ & - & $67 \pm 30$ & $8.14 \pm 0.04$ & $f(0.01)$ & $-54 \pm 20$ & $0.94 / 627$ \\
\hline $\operatorname{MOS} 1+2$ & PL & $2.34 \pm 0.05$ & - & - & - & - & - & - & $1.199 / 257$ \\
\hline $\operatorname{MOS} 1+2$ & $\mathrm{PL}+\mathrm{NGL}$ & $2.36 \pm 0.05$ & $7.04_{-0.11}^{+0.09}$ & $f(0.1)$ & $81_{-43}^{+49}$ & - & - & - & $1.171 / 255$ \\
\hline MOS1+2 & PL+2 NGLs & $2.38 \pm 0.05$ & $f(7.04)$ & $f(0.1)$ & $83 \pm 46$ & $6.64_{-0.28}^{+0.22}$ & $f(0.1)$ & $38 \pm 35$ & $1.163 / 254$ \\
\hline $\operatorname{MOS} 1+2$ & $\mathrm{PL}+\mathrm{BGL}$ & $2.38 \pm 0.05$ & $6.89 \pm 0.26$ & $0.29_{-0.11}^{+0.26}$ & $138_{-70}^{+54}$ & - & - & - & $1.172 / 254$ \\
\hline $\operatorname{MOS} 1+2$ & $\mathrm{PL}+\mathrm{DL}$ & $2.39 \pm 0.05$ & $6.76 \pm 0.10$ & - & $158_{-77}^{+71}$ & - & - & - & $1.159 / 255$ \\
\hline $\operatorname{MOS} 1+2$ & $\mathrm{PL}+\mathrm{DL}+\mathrm{ABL}$ & $2.37 \pm 0.05$ & $6.76 \pm 0.11$ & - & $141_{-72}^{+75}$ & $7.91 \pm 0.08$ & $f(0.01)$ & $-62 \pm 42$ & $1.146 / 253$ \\
\hline
\end{tabular}

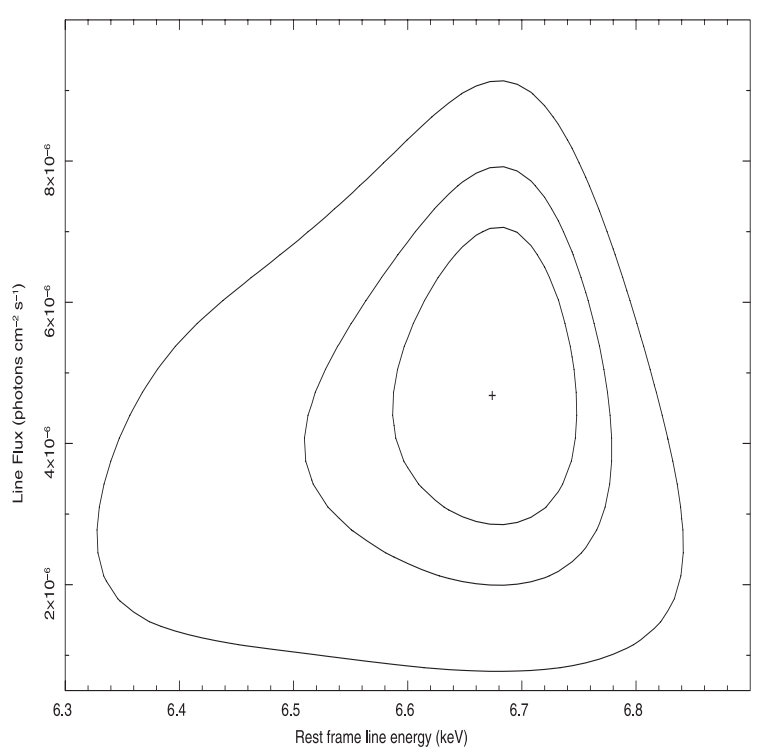

Fig. 4. Plot of the $68 \%, 90 \%$, and $99 \%$ confidence level contours (from the inner to the outer curves, respectively) for the rest-frame energy and the line flux of the iron emission line in the case of the PL+NGL best fitting model to the EPIC PN data.

in Table 1 (model "PL+DL"). The goodness of fit is similar to that of the PL+NGL model, and the best best-fitting line energies are similar in the two models.

We conclude that there is no significant evidence for the presence of a broad iron emission line in the EPIC PN data. In Fig. 4 we show the confidence contour plot for the restframe energy and intensity of the detected line in the case of the PL+NGL model. The contours plotted correspond to the $68 \%$, $90 \%$, and $99 \%$ confidence levels (from the inner to the outer curves) for two interesting parameters.

In order to assess the importance of the absorption feature at $8 \mathrm{keV}$, we added a narrow Gaussian line (i.e. with $\sigma$ kept fixed at $10 \mathrm{eV}$ ) in the PL+DL model and forced its normalization to have negative values only (model "PL+DL+ABL" in Table 1). Compared to the best fitting PL+DL model, this addition improved the goodness of fit by $\Delta \chi^{2}=17.7$ for 2 d.o.f., which is highly significant. The best-fitting absorption line's energy is $\sim 8.1 \mathrm{keV}$, and its equivalent width is $\sim-60 \mathrm{eV}$. Figure 5 shows the best fitting PL+DL+ABL plot to the PN. The residuals plot (lower panel in the same figure) shows no clear systematic

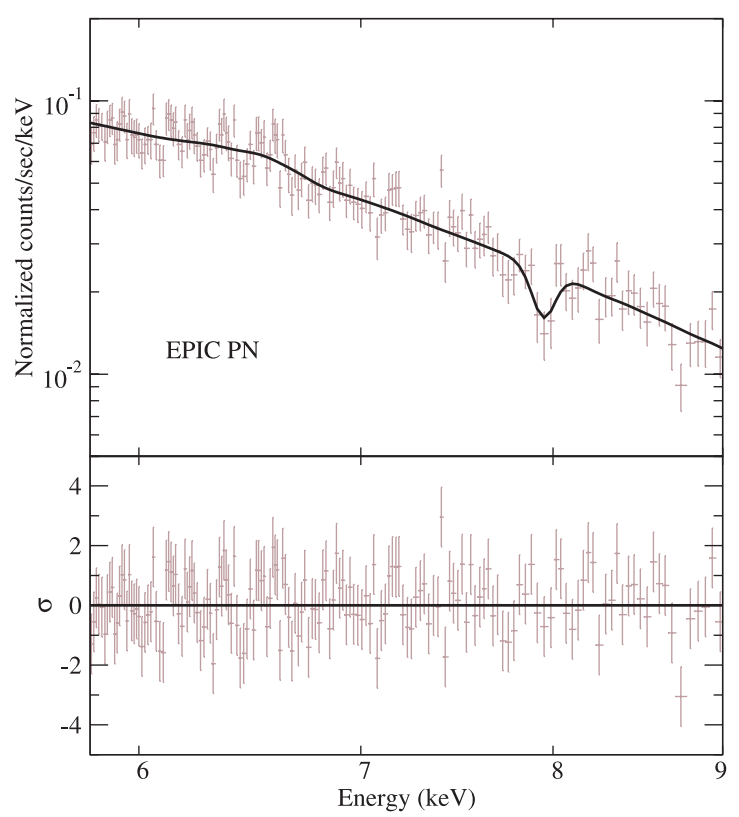

Fig. 5. "Power-law plus discline plus absorption line" best fitting model plot to the PN data (upper panel) and residuals plot (lower panel) in the $5.8-9 \mathrm{keV}$ energy band.

deviations from this model. The model residuals in the region between 6-6.5 keV are somehow "noisy", but they are almost certainly caused by local statistical fluctuations. Further attempts to model them with extra narrow emission or absorption components did not lead to any reasonable results.

\subsection{The hard band EPIC MOS energy spectra}

Figure 6 shows the best-fitting PL model fit to the MOS1 and 2 data and the corresponding residuals in the 3-11 keV band (top and middle panels, respectively). Clearly, the MOS 1 and MOS 2 spectra in the hard band are similar. For that reason, and in order to increase the signal to noise ratio, we added them together and created the combined "MOS $1+2$ " spectrum. The bottom panel in the same figure shows the residuals in the case of the best-fitting PL model to the MOS1+2 spectrum. The residuals plot is rather noisy but a PL model appears to fit the overall spectrum well. The main residual feature is a low-amplitude excess emission around $\sim 6.5-7 \mathrm{keV}$. 


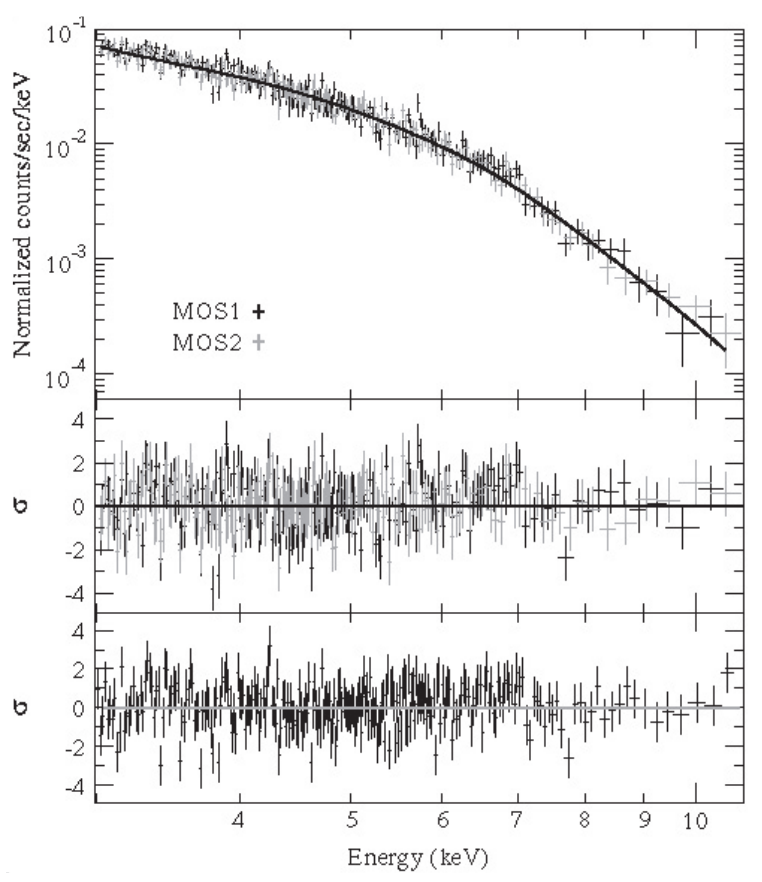

Fig. 6. Plot of the power-law fit (upper panel) and residuals (middle panel) to the joint MOS 1 and 2 data in the $3-11 \mathrm{keV}$ energy band. In the bottom panel we plot the best power-law fit residuals in the case of the combined MOS1+2 spectrum. Notice the residuals in the Fe line region and the absorption feature around $\sim 7.8 \mathrm{keV}$, just like in the case of the PN spectrum.

We fitted the MOS1+2 spectrum with the same models that we used in the case of the PN spectrum. The best fitting results are listed in Table 1 and are broadly consistent with those we reached from the PN spectral analysis. Compared with the best fitting PL model, the addition of either a narrow, or a broad Gaussian line, or a DISKLINE component improves the quality of the fit at the 98.1,96.6 and 99.5\% levels, respectively. Compared to the best fitting PL+NGL model, neither the addition of a second narrow Gaussian line nor the use of the PL+BGL model improves the fit significantly.

There is a hint of an absorption line at $7.9 \mathrm{keV}$ in the MOS1+2 spectrum, but the statistics of the MOS data are not sufficient to confirm or refute the absorption line that appears in the PN data at $8.1 \mathrm{keV}$. Indeed, the improvement to the goodness of fit when we add an absorption line to the PL+DL model is not significant (it improves the fit by $91.3 \%$ ). This is not surprising given the poorer statistics of the MOS spectra with respect to the PN spectrum. However, note that, among all the models listed in Table 1, only the PL+DL+ADL model fits the MOS1+2 spectrum at better than the $5 \%$ confidence level.

As commonly found, the fitted power-law slopes for the MOS are slightly flatter than those for the PN by $\Delta \Gamma \sim 0.08-0.1$, for all models listed in Table 1. Furthermore, the MOS1+2 best fitting line centroid energies are systematically higher, and the line's $E W$ larger, than those resulting from the PN model fitting. If we consider the PL+DL+ABL model, for which the differences in the PN and MOS1+2 best model fitting parameter values are the smallest, then the average $\mathrm{PN}+\mathrm{MOS}$ spectral slope is $\Gamma=2.43 \pm 0.03$, the average iron emission line energy is $6.67 \pm$ $0.07 \mathrm{keV}$, and its equivalent width is $78 \pm 28 \mathrm{eV}$.

We conclude that both the PN and MOS 3-11 keV band spectra are well fitted by a power-law model of slope $\Gamma \sim 2.43$. There is significant evidence for an iron emission line at $6.7 \mathrm{keV}$

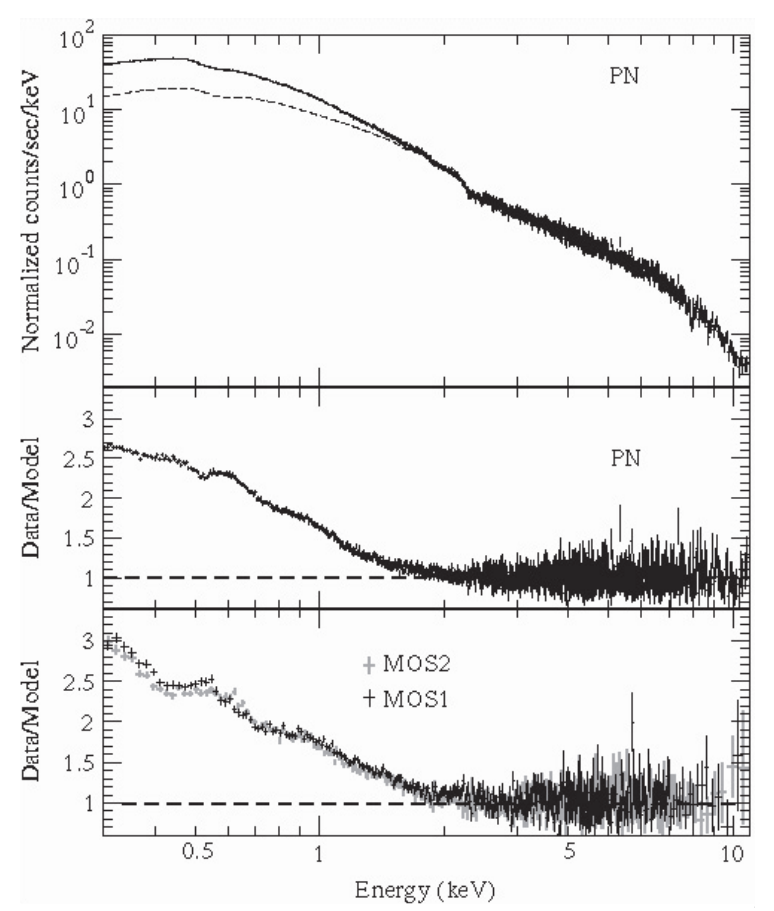

Fig. 7. Extrapolation of the hard band, best fitting "Pl+DL+ABL" model into the soft band to demonstrate the soft excess. The upper two panels show the full-band data, the hard band best model fit and the data-to-model ratio for the PN data, while the lower panel shows the ratios for the two MOS spectra.

which implies emission from ionized iron. We find no evidence for an extra narrow line emission component either at lower or higher energies. The line is weak $(E W \sim 80 \mathrm{eV})$ and narrow. Although models like a broad Gaussian and a DISKLINE do fit the data well, they are not statistically required. Finally, a narrow absorption feature at $8.1 \mathrm{keV}$ appears in the PN spectrum.

\subsection{The broad band EPIC spectra}

An extrapolation of the best fitting PL+DL+ABL model to low energies shows a broad excess of emission, both in the PN and the two MOS spectra (see Fig. 7). The lower panels in Fig. 7 show the data/model residuals for the PN and MOS spectra. The smooth, extra component which dominates the source's emission at energies below $2 \mathrm{keV}$ is clearly seen. There appear no strong spectral features, either in emission or absorption, while the soft excess component flattens at energies below $\sim 0.5 \mathrm{keV}$.

The nature of the small amplitude structures that we observe might be intrinsic to the source but remaining calibration uncertainties cannot be ruled out either, considering the excellent signal to noise that we have achieved at low energies in this long observation. For example, the obvious structures at $\sim 0.5 \mathrm{keV}$ are most probably caused by calibration uncertainties. Furthermore, the MOS 1 and 2 spectra, although quite similar at energies above $\sim 0.6 \mathrm{keV}$, deviate significantly at lower energies. Clearly, this also reflects remaining calibration uncertainties in the MOS detectors at low energies.

In order to minimize the influence of the instrumental response and gain some insight into the intrinsic broad-band shape of the source spectrum, the PN data of Ark 564 were compared to the PN spectrum of 3C 273. Specifically, we divided the PN raw spectrum by the raw spectrum of a $\sim 18$ ks observation of 3C 273 from June 30, 2004, taken in the same SW mode with 


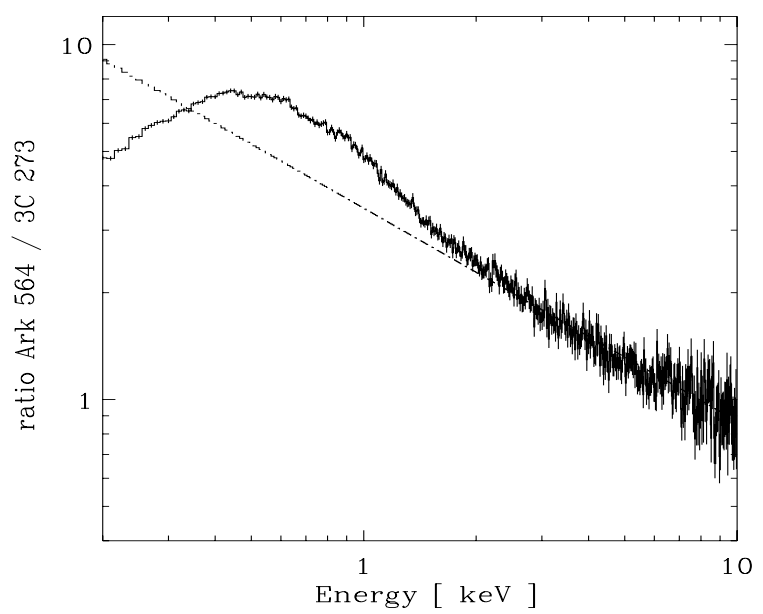

Fig. 8. Ratio of the raw PN counts of Ark 564 divided by the raw PN counts of 3C 273. The dash-dotted line is a power-law approximation to the hard part of the spectrum with a slope of $\Gamma=0.6$ representing the difference of the slopes of the hard spectra of the two sources.

medium filter. We chose 3C 273 as it is a bright source with a relatively simple spectrum in the EPIC band, i.e. it shows a hard power-law plus smooth soft excess modified by Galactic absorption, without strong, sharp spectral features such as edges (Page et al. 2004). As a result, the ratio of the Ark 564 to the 3C 273 spectrum will factor out most effects of the instrumental response and will give a better view of the intrinsic shape of the Ark 564 spectrum.

In Fig. 8 we display this ratio. The straight dash-dotted line indicates the difference in the slopes of the intrinsic hard band power laws of the two objects. The downward bending of the ratio at energies below $\sim 0.4 \mathrm{keV}$ can be attributed to the smaller amount of absorption for 3C $273\left(N_{\mathrm{H}} \sim 1.69 \times 10^{20} \mathrm{~cm}^{-2}\right)$. The ratio shows some small amplitude "wiggles" but by no means any prominent spectral structures. For example, Vaughan \& Fabian (2004) have followed a similar approach, and show in their Fig. 3 the ratio of the raw MCG -6-30-15 PN spectrum to the raw PN spectrum of $3 \mathrm{C} 273$. We do not observe any of the strong absorption features that appear in MCG -6-30-15 at low energies. In fact, the ratio plot shown in Fig. 8 suggests that both the existence of strong absorption edges and/or emission lines in the $\sim 0.5-1.5 \mathrm{keV}$ band is rather unlikely. Furthermore, the ratio behaves smoothly even at the lowest energies (down to $0.2 \mathrm{keV}$ ), in contrast to the results from the spectral fits (to the MOS spectra mainly) that we present in the following sections.

\subsection{Describing the soft excess}

We tried to model the soft excess with a variety of different models to understand its physical nature. To this end, we made the following assumptions. As with the hard band model fits, the Galactic absorption was modeled using PHABS with $N_{\mathrm{H}}=6.4 \times$ $10^{20} \mathrm{~cm}^{-2}$ and the abundance table of Lodders (2003). We also tried the tables of Grevesse \& Sauval (1998) and Wilms et al. (2000), but their use resulted in systematically worse model fits (for the adopted $N_{\mathrm{H}}$ value).

For the PN spectrum, we used the best fitting PL+DL+ABL model as the "baseline" model to which various components were added in order to model the soft excess. The reason for this choice is not based on physical considerations (i.e. the iron emission feature in the hard band PN spectrum is equally well fitted by a narrow Gaussian line)
Table 2. Results from the spectral fitting in the $0.3-11 \mathrm{keV}$ energy band assuming fixed Galactic $N_{\mathrm{H}}=6.4 \times 10^{20} \mathrm{~cm}^{-2}$.

\begin{tabular}{lcc}
\hline \hline Model & $\chi_{\text {red }}^{2} /$ d.o.f. $(\mathrm{PN})$ & $\chi_{\text {red }}^{2} /$ d.o.f. $(M O S)$ \\
\hline BASELINE+PL & $11.85 / 1174$ & $2.66 / 753$ \\
BKN PL & $4.84 / 1173$ & $1.98 / 758$ \\
2 BKN PL & $1.52 / 1171$ & $1.66 / 756$ \\
BASELINE+BB & $4.88 / 1173$ & $4.76 / 753$ \\
BASELINE+2 BBs & $1.29 / 1171$ & $1.55 / 751$ \\
BASELINE+BREMS & $1.35 / 1173$ & $1.81 / 753$ \\
\hline
\end{tabular}

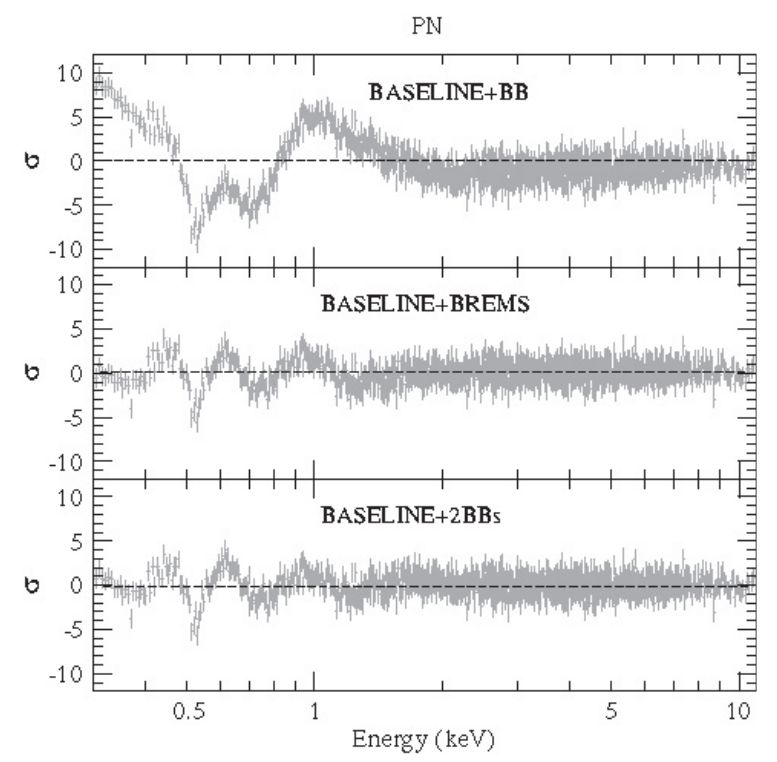

Fig. 9. Best model fit residuals to the full band PN energy spectrum of Ark 564.

but simply on the fact that this model yields the smallest best fitting $\chi^{2}$ value, in the hard band, among all the models listed in Table 1 . During the model fitting, the model parameter values were kept fixed to the values listed in Table 1, except for the PL normalization which was left as a free parameter.

The models we employed for the soft band spectrum were the following: 1) a soft band power-law ("BASELINE+PL"), 2) a break in the hard power law ("BKN PL"), 3) two breaks in the hard power-law ("2 BKN PL"), 4) a blackbody ("BASELINE+BB"), 5) two blackbodies ("BASELINE+2BBs"), and 6) a thermal bremsstrahlung spectrum ("BASELINE+BREMS"). None of them resulted in statistically acceptable fits. In Table 2 we list the $\chi_{\text {red }}^{2} /$ d.o.f. for each model, and in Fig. 9 we show the best fitting model residuals in the case of the most favorable model fits to the PN spectrum (BASELINE+2 BBs and BASELINE+BREMS). For comparison reasons, we also show the best fitting residuals in the case of the BASELINE+BB model.

Despite the formally unacceptable large $\chi_{\text {red }}^{2}$ values, the plots in Fig. 9 suggest that the BASELINE+2BBs and BASELINE+BREMS models provide reasonable fits to the broad band PN spectrum. In the case of the BASELINE+BREMS model we find $k T \sim 0.3 \mathrm{keV}$. The best-fitting temperatures of the two blackbodies are $\sim 0.08$ and $\sim 0.16 \mathrm{keV}$ (since the models do not give acceptable fits, error estimates of the model parameters are not meaningful).

The models listed in Table 2 were also fitted to the MOS 1 and 2 spectra. In this case, we did not add the two MOS spectra together, due to their differences at low energies. Instead, 


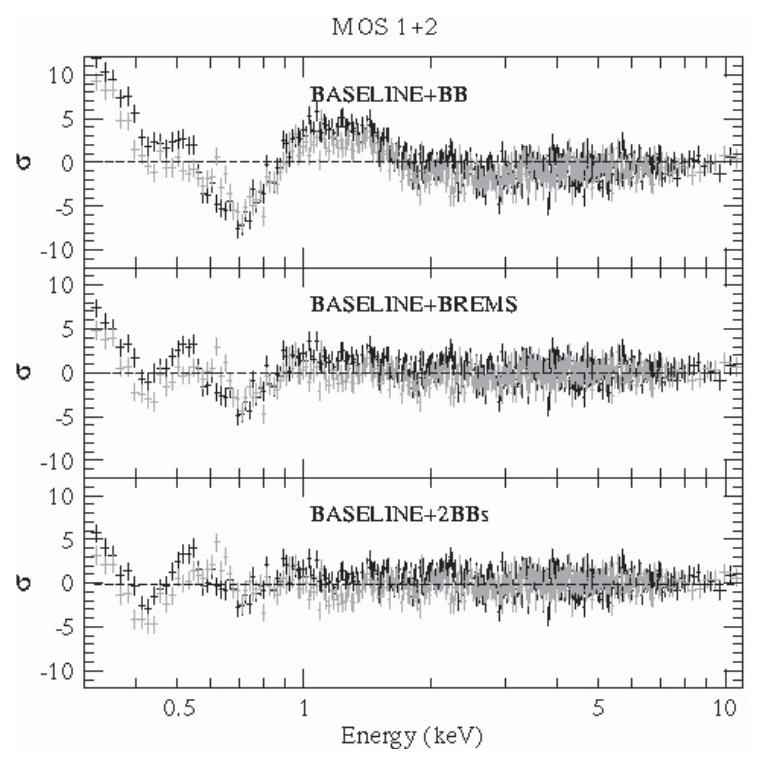

Fig. 10. Best model fit residuals to the full band MOS 1 and 2 energy spectra of Ark 564.

we performed joint model fits to both of them, and we plot the residuals to the best fit model for both detectors. The values of $\chi_{\text {red }}^{2}$ for these model fits are listed in the last column of Table 2 and residual plots for a few models are shown in Fig. 10. As with the PN spectrum, none of the models provide a statistically acceptable fit to the data. The BASELINE+2 BBs and BASELINE+BREMS models provide again reasonable fits to the MOS spectra. The best fitting parameter values $(k T \sim 0.37 \mathrm{keV}$, in the case of the BASELINE+BREMS model, and blackbody temperatures of $\sim 0.08$ and $0.19 \mathrm{keV}$ ) are consistent with the results from the PN spectrum.

A comparison between the respective plots in Figs. 9 and 10 shows that the residual structures are similar in all detectors. The use of a single black body component always gives rise to a broad excess bump in the $0.8-1.5 \mathrm{keV}$ range and deep, edgelike structures, between $\sim 0.5$ and $0.8 \mathrm{keV}$. Adopting a thermal bremsstrahlung or two black body components considerably improves the fit, and minimizes the residuals around the $1 \mathrm{keV}$ and $0.5-0.8 \mathrm{keV}$ features. At energies below $0.6 \mathrm{keV}$, the MOS 1 and 2 residuals are different from each other and from those seen in the PN spectra. These discrepancies must be attributed to calibration uncertainties of the instruments.

Clearly, as the reduced $\chi^{2}$ values demonstrate, an ideal bremsstrahlung or two black body components are not exact models for the soft excess. However, the deviations of the observed spectrum from the best fitting model shapes are only at a few percent level (see top panel in Fig. 12) and they could reflect to a large extent remaining calibration uncertainties. For example, the most prominent residual features in the PN best fitting BASELINE+2 BBs and BASELINE+BREMS models are around $\sim 0.5 \mathrm{keV}$ (where we expect the instrumental and Galactic oxygen edges to appear). However, the other residual features around $0.7-0.8 \mathrm{keV}$, where a broad, shallow deficit appears, and $\sim 0.9-1 \mathrm{keV}$, where we observe an excess above the best fitting model could correspond to low amplitude, absorption features intrinsic to the source. This possibility can be investigated with the study of RGS spectrum, which we describe below.

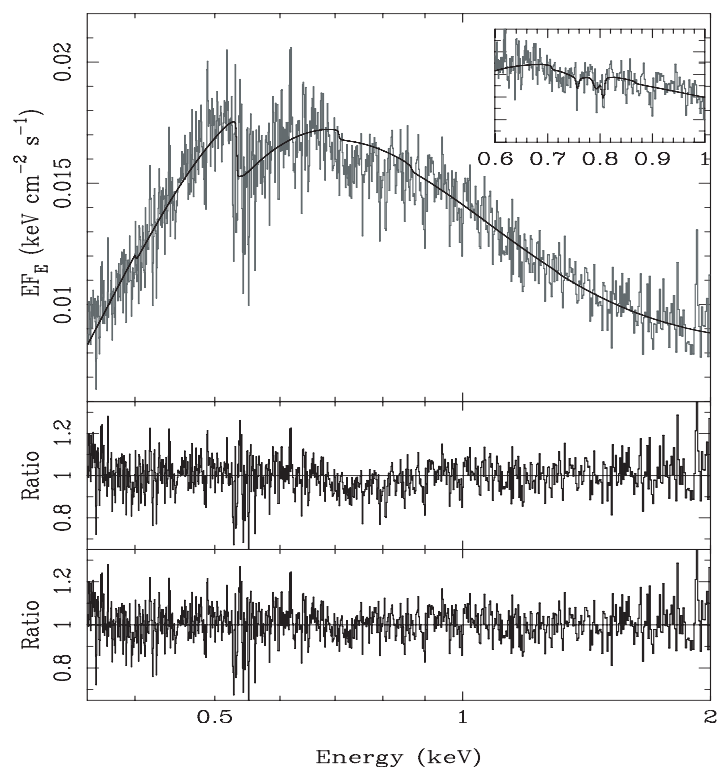

Fig. 11. Top panel: the RGS spectrum of Ark 564 and the best fitting continuum model (black line) in the $<0.6$ and $>0.9 \mathrm{keV}$ band. The spectrum shown is fluxed (i.e. the data points are divided by the effective area) but not unfolded. Inset: close up of the $0.6-1 \mathrm{keV}$ region with absorption lines added to the model to represent the $0.7-0.9 \mathrm{keV}$ Fe UTA features. Middle panel: data/model ratio for the continuum model fit in the same energy band. Bottom panel: data/model ratio when absorption lines are included in the model to represent the $0.7-0.9 \mathrm{keV}$ Fe UTA feature.

\subsubsection{The RGS data}

Due to the EPIC-RGS cross calibration problems, and the timedependent degradation of the RGS low-energy effective area, which is not yet accounted for in the SAS (Stuhlinger et al. 2006), the RGS data cannot be compared directly to the best fitting EPIC models. However, this is not a serious problem, as our main aim in this work is to identify any strong emission and/or absorption features in the RGS spectra that may affect the goodness of the various model fits to the EPIC spectra. To this end, all we need is to model the continuum in the RGS spectrum as accurately as possible and search for any remaining residuals.

We therefore used the BASELINE+2BBs model to fit the RGS spectrum. The power-law and blackbody model parameters were left to vary as free parameters and the Galactic absorption was modeled as in the case of the EPIC spectra. The best-fitting RGS power-law index is harder than that found with $\mathrm{PN}(\Delta \Gamma \sim-0.5)$, as expected with the current RGS/EPIC crosscalibration (Stuhlinger et al. 2006). The two blackbodies have best fitting $k T$ of $0.1 \mathrm{keV}$ and $0.2 \mathrm{keV}$, similar to those determined with the PN.

The model fits the overall shape of the RGS spectrum well over the $0.35-2 \mathrm{keV}$ range, except for some strong residuals around the Galactic O I edge (as with the EPIC spectra), a number of narrow absorption lines, and a broad deficit of flux in the $0.65-0.85 \mathrm{keV}$ range. Interestingly, this residual feature is almost identical to a similar deficit that appears in the EPIC residuals plot as well (Figs. 9 and 10). The RGS spectrum, the continuum model (fitted to the data below $0.6 \mathrm{keV}$ and above $0.9 \mathrm{keV}$ ), and the data/model ratio are shown in the top and middle panels of Fig. 11, respectively. The location of the broad dip centered at $0.77 \mathrm{keV}$ corresponds to the blended unresolved transition arrays of Fe I-XVI. A detailed study of this feature and of the narrow absorption lines in the Ark 564 RGS spectrum, using the 
sophisticated warm absorber models available in the SPEX spectral fitting package, will be presented elsewhere (Smith et al. in preparation). For the purposes of this work, in order to represent the absorption in the Fe UTA region, we added 4 Gaussian absorption lines to the continuum model and fitted again the spectrum using XSPEC.

The results are shown in the inset panel of Fig. 11, while the data/model ratio is plotted in the bottom panel of the same figure. The model now fits well the overall shape of the entire spectrum, but the statistical goodness of fit is still poor $\left(\chi_{\text {red }}^{2} /\right.$ d.o.f. $=$ $1.97 / 922)$, primarily because of the structure around the Galactic O I edge, and other narrow absorption features in the spectrum. Although the remaining absorption lines and features in the RGS spectrum of Ark 564 outside the Fe UTA region are significant, they are too weak to warrant inclusion in the EPIC spectral model.

In a previous study of the X-ray spectrum of Ark 564 using two short XMM-Newton observations, Vignali et al. (2004) included a strong photoelectric edge from O VII in their spectral model. Such an edge, which has a threshold energy of $0.72 \mathrm{keV}$, is not visible in the RGS spectrum (Fig. 11). Replacing the UTA features in our spectral model with an O VII edge yields $\chi_{\text {red }}^{2} /$ d.o.f. of $2.02 / 927$, a poorer fit by $\Delta \chi^{2}=46$ with respect to our best fit model. The fitted optical depth of the edge is $\tau=0.06 \pm 0.02$, much smaller than the $\tau=0.4 \pm 0.1$ reported by Vignali et al. (2004), but similar to the optical depth determined by Matsumoto et al. (2004) in their HETGS observation $\left(\tau=0.07_{-0.05}^{+0.04}\right)$. If both the edge and the 4-Gaussian representation of the Fe UTA features are included in the spectral model we obtain $\chi_{\text {red }}^{2}$ /d.o.f. of $1.97 / 921$, no better than the model without the edge. In this case the O VII edge is insignificant, with $\tau<0.06$ ( $3 \sigma$ limit).

We conclude that the soft band spectrum of Ark 564 is indeed intrinsically smooth. The most significant spectral feature appears in the $0.7-0.9 \mathrm{keV}$ band, and most probably corresponds to the blended unresolved transition arrays of Fe I-XVI. In particular, we do not observe any strong absorption edges around $0.7 \mathrm{keV}$ or any emission features around $0.9-1.1 \mathrm{keV}$.

\subsubsection{The EPIC PN spectrum revisited}

In the top panel of Fig. 12 we show the data/model ratio in the case of the BASELINE+2 BBs model fits to the PN spectrum (for clarity reasons, in Fig. 12 we show the data/model ratio over the softest band only). As we mentioned earlier, the deviations are at the $\$ 4 \%$ level (over the whole energy band). Since the results from the RGS spectral analysis have shown that the broad, shallow deficit of flux in the $0.65-0.85 \mathrm{keV}$ range is most probably intrinsic, we fitted again the EPIC PN spectrum with the BASELINE+2BBs and BASELINE+BREMS models, including the 4 Gaussian lines that we used to model the absorption in the Fe UTA region. Their centroid energy, width and normalization were kept fixed at the values we got from the RGS model fitting. The addition of these 4 lines yields $\chi_{\text {red }}^{2}$ /d.o.f. of $1.22 / 1171$ and $1.36 / 1173$, respectively. Compared to the simple BASELINE+BREMS model, the new model fit is poorer by $\Delta \chi^{2}=12.2$. On the other hand, the addition of the absorption lines representing the Fe UTA to the simple BASELINE+2 BBs model, improves the model fit by $\Delta \chi^{2}=74.7$, with no additional degrees of freedom.

This result demonstrates that the large $\chi_{\text {red }}^{2}$ values associated with the models listed in Table 2 are due, to a large extent, to the presence of warm absorber features. Indeed, in the middle panel of Fig. 12 we show the data/model ratio in the case of the

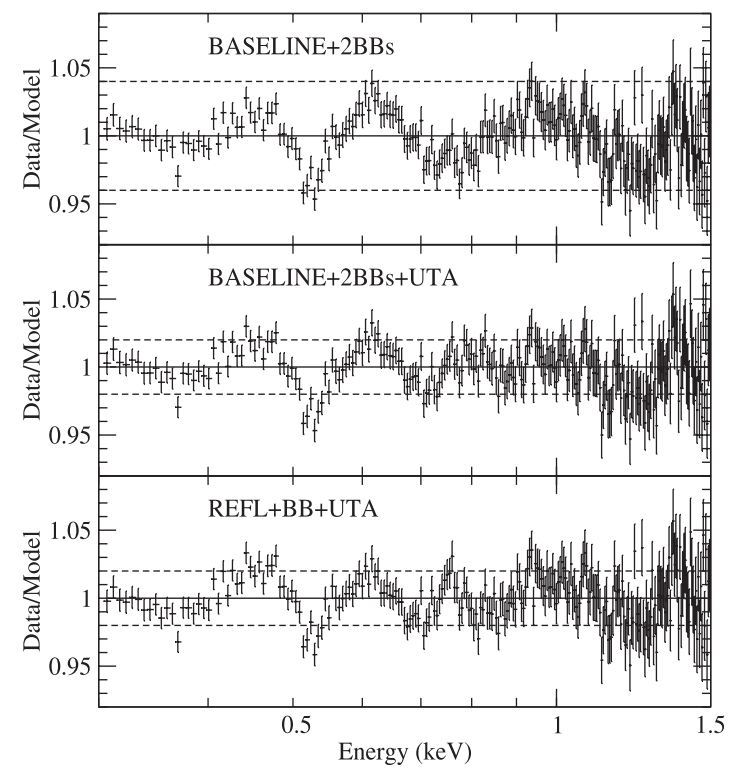

Fig. 12. Data to model ratio of the "BASELINE+2BBs" (top panel), the "BASELINE+2BBs+UTA" (middle panel) and the "REFL+BB+UTA" model fits to the PN data in the $0.3-1.5 \mathrm{keV}$ band. For comparison reasons, the $y$-axis is identical in both panels.

BASELINE+2 BBs+UTA model. The model deviations are now reduced to $\lesssim$ the $2 \%$ level over almost the entire energy band.

However, the best fitting kT parameter values of the two black body components are $\sim 0.15 \mathrm{keV}$ and $\sim 0.07 \mathrm{keV}$. These temperatures are rather high and difficult to explain in terms of simple accretion disc predictions (see Sect. 5). For these reasons, we furthermore examined two alternative models which are physically more motivated and have already been used to model the soft excess successfully in a few AGN.

The first one involves Comptonization. A two-temperature distribution of hot electrons can in principle lead to the formation of both the hard power-law and the soft excess. In order to investigate this possibility we fitted the broad band spectrum of Ark 564 with the BASELINE plus a COMPTT component (Titarchuk 1994) to account for the soft excess, a black body component (to account for intrinsic emission from the innermost disc) and the 4 narrow Gaussian lines for the UTA absorption feature. This model does not provide a good fit to the full band spectrum of Ark 564 ( $\chi_{\text {red }}^{2}=1.89 / 1167$ d.o.f. $)$, with large amplitude residuals appearing in the soft band.

An alternative possibility is that the soft excess emission is due to reprocessing of the primary X-rays in the accretion disc. If the disc surface is highly ionized, a significant excess of emission is expected to emerge in the soft X-ray band, including strong emission lines from the irradiated disc. If the emission features are smeared out by the motion of the disc, or by general relativistic effects, then the soft excess emission could appear smooth. In order to investigate this possibility, we used the ionized-reflection model REFLION of Ross \& Fabian (2005) in XSPEC together with a power-law component (to account for the illuminating source). In order to take into account the Doppler and gravitational effects around a black hole we adopted a simplified approach and convolved the emitted spectrum with a Gaussian of $v=0.2 \mathrm{c}$ width. We also added the narrow Gaussian line for the absorption feature at $8.1 \mathrm{keV}$, a black body component, and the four narrow Gaussian absorption lines at $~ 0.75-0.8 \mathrm{keV}$ ("REFL+BB+UTA" model). 
The model fits the broad band spectrum as well as the BASELINE+2 BBs+UTA model $\left(\chi_{\text {red }}^{2}=1.23 / 1169\right.$ d.o.f. $)$. In the bottom panel of Fig. 12 we show the data/model ratio in this case. The best fit model parameters are $\Gamma \sim 2.46, k T \sim 0.08 \mathrm{keV}$ for the black body temperature, iron abundance $\sim$ solar, and ionization parameter $\xi^{1} \sim 2000 \mathrm{erg} \mathrm{cm} \mathrm{s}{ }^{-1}$. The measured (unabsorbed) ratio of the reflected flux to the total observed flux is $\sim 0.45$.

For both models, the largest amplitude residuals appear around the Galactic O I edge. In order to investigate this issue further, we repeated the model fits with the $N_{\mathrm{H}}$ and the oxygen abundance parameters set free to vary during the fitting process. We found no significant improvement to the previous model fitting results. Most probably, the residuals around $\sim 0.5-0.6 \mathrm{keV}$ are caused by the current uncertainties associated with the proper calibration of the instrumental and Galactic feature at $0.543 \mathrm{keV}$ in the PN.

With the Fe UTA features included in the PN fit, there is little evidence for an emission line at around $1 \mathrm{keV}$. Nevertheless, in order to investigate this issue further, we added a narrow Gaussian line component (with $\sigma$ fixed at $5 \mathrm{eV}$ ) in the BASELINE+2 BBs+UTA model and fitted again the PN spectrum. The resulting line has a centroid energy of $\sim 0.96 \mathrm{keV}$ and an equivalent width of $\sim 1 \mathrm{eV}$. However, we get an improvement in the goodness of fit by $\Delta \chi^{2}=6.2$ for 2 additional degrees of freedom, which is not significant.

We conclude that, when we consider the UTA absorption features in the 0.7-0.8 keV band, both the BASELINE+2 BBs and REFL+BB+UTA models fit well the full band X-ray spectrum of Ark 564. The fits are not formally acceptable. However, the magnitude of the data/model residuals is comparable to the accuracy with which the PN calibration is currently known. This result, together with the "smoothness" of the Ark 564/3C 273 PN spectrum (Fig. 8), suggest that the statistically significant discrepancies found between the data and the two models may well reflect, to a large extent, remaining calibration uncertainties.

\section{Discussion}

Spectral variability. As we mentioned in Sect. 3, the large amplitude flux variations of Ark 564 are associated with spectral variations as well. Changes in the continuum spectral shape can provide us with important clues regarding the nature of the physical mechanism responsible for the X-ray emission in the source. Furthermore, time-average spectral analysis can miss valuable information from the temporal behaviour of the emission/absorption features in the spectrum.

We plan to study in detail the spectral variability properties of the source in the near future (Brinkmann et al., in preparation). Nevertheless, in order to give an idea of how the spectral characteristics of the source change with time, we generated two EPIC PN spectra (with a minimum of 30 counts/bin as for the time-average spectrum) for the time periods between $10-15 \mathrm{ks}$ and $67-70 \mathrm{ks}$ after the start of the observation. As can be seen from Fig. 1, these spectra correspond to time periods when the hardness ratio was low and high (we call them as the HRL and HRH spectra, respectively). In both cases, the $3-11 \mathrm{keV}$ spectra are well fitted by a simple PL model $\left(\Gamma_{\mathrm{HRL}}=2.53 \pm\right.$ $0.11, \Gamma_{\mathrm{HRH}}=2.25 \pm 0.12$, and $\chi_{\text {red,HRL }}^{2}=0.96 / 103$ d.o.f., $\chi_{\text {red,HRH }}^{2}=0.94 / 74$ d.o.f.). The full band spectra can be well

${ }^{1} \xi=L / n r^{2}$, where $L$ is the hydrogen ionizing luminosity, $n$ is the density, and $r$ is the distance between the absorber and the source of ionization radiation. fitted by the BASELINE+2BBs model $\left(\chi_{\mathrm{HRL}}^{2}=557.8 / 537\right.$, and $\chi_{\mathrm{HRH}}^{2}=505.4 / 468$ d.o.f. $)$.

We found no indication of any emission and/or absorption features at $\sim 6.5-7 \mathrm{keV}$ and $\sim 8 \mathrm{keV}$, and we could not detect the UTA feature in the soft band, in either of the two spectra. This is not surprising, given the small amplitude of these features in the time-average spectrum of the source. Consequently, these features can be studied best with the use of the time-average spectrum (which we present in this work), since, due to their weakness, they can hardly be detected in spectra which are extracted from shorter time periods.

The best fitting kT values in the case of the HRL spectrum are $0.16 \pm 0.01$ and $8.1 \pm 0.5 \times 10^{-2} \mathrm{keV}$. The respective values in the case of the HRH spectrum are $0.17 \pm 0.01$ and $7.7 \pm$ $0.3 \times 10^{-2} \mathrm{keV}$. Thus, although the soft component's flux varied between the two periods, its shape remained almost the same. In fact, Brinkmann et al. show that the shape of this component remains roughly constant throughout the present XMM-Newton observation. Hence, the spectral shape of the soft excess component can also be studied best with the time-average spectrum, as in this case we take advantage of the highest possible signal-tonoise ratio data.

As for the hard band power-law component, the PL model fits to the HRL and HRH spectra suggest a spectral slope variation of the order of $\Delta \Gamma \sim 0.3$, which is certainly not negligible. The results from a detailed study of the spectral slope variability will be presented in a forthcoming paper by Brinkmann et al. For the time being, we can think of the slope of the time-average spectrum as a measure of the average spectral slope during the XMM-Newton observation of Ark 564.

The hard band continuum. A power-law model, with $\Gamma=$ $2.43 \pm 0.03$ fits well both the PN and MOS time-average spectra at energies above $3 \mathrm{keV}$. This is in excellent agreement with the results from previous observations of Ark 564.

This slope is quite steep even for a NLS1 (Leighly 1999b). It is interesting to compare Ark 564 with the Galactic black hole X-ray binaries (BHXBs) since it is widely believed that the physical processes that operate in AGN and BHXBs are similar. BHXBs exhibit photon indices steeper than 2.4 only when they are in the so-called "Very High" (VH) or "Steep PowerLaw" (SPL) spectral state (McClintock \& Remillard 2005). In this state, black hole binaries are usually quite bright, with accretion rates higher than $\sim 0.2$ in Eddington units, exactly like Ark 564 which is also believed to be a high accretion rate system (e.g. Romano et al. 2004). One possible explanation for the various BHXB spectral states invokes changes in the accretion flow geometry. For example, Done (2002) argues in favour of the inner radius of the accretion disc decreasing with increasing accretion rate. At high accretion rates (like in NLS1s), the disc could even extend down to the last stable orbit. At the same time, Merloni (2003) argues that, if the viscosity parameter is large, high accretion rates are accompanied by powerful, magnetically dominated coronae. Because of the changing disc geometry and the high accretion rate, the density of soft photons is high, leading to a more efficient cooling of the corona. Consequently, the resultant Comptonized spectrum from the corona becomes soft. In other words, the consistently steep hard band power-law in Ark 564 implies that the geometry of the accretion disc and/or the corona may be different to that in the "classical" Seyfert galaxies.

The iron emission line. We detect a significant iron line emission feature at $\sim 6.7 \mathrm{keV}$, in both the PN and MOS spectra. The line is weak $(E W \sim 80 \mathrm{eV})$ and can be fitted well with a narrow Gaussian line. It can also be fitted well by a broad Gaussian line 
or a DISKLINE model, but the use of these models is not statistically required. In fact, the limited data quality in the iron line region due to its weakness prevents a more precise determination of the line parameters.

Our results are in agreement with those of Vaughan et al. (1999) who also detected a rather weak, (i.e. $E W \sim 95 \mathrm{eV}$ ), narrow, iron line in the ASCA and RXTE combined spectrum they studied. However, they are not in agreement with those of Turner et al. (2001) who detected a very strong $(E W \sim 650 \mathrm{eV})$ iron emission line during the one month long ASCA monitoring in 2000 , despite the fact that the continuum slope and luminosity (above $3 \mathrm{keV}$ ) are comparable in both cases. Therefore, variability in the line strength due to differences in the primary radiation cannot account for the observed difference in the line properties. The rather unusual magnitude of the previously reported feature, and the low signal to noise ratio at the high energy end of a steep spectrum, seem to point toward background subtraction problems in the earlier data.

On the other hand, the line's energy is in agreement with the $A S C A$ results, and suggests the presence of highly ionized material. The presence of a line indicative of highly ionized iron is not surprising in the case of Ark 564, since the incident X-ray spectrum is quite steep. In this case, the Compton temperature even on the top of the layer responsible for the X-ray reflection can be lower than $\sim 1 \mathrm{keV}$. This allows some of the highest ionization stages of iron to be abundant enough to imprint highly ionized signatures on the X-ray spectrum, i.e. lines at 6.7 or $6.9 \mathrm{keV}$ (Nayakshin et al. 2000).

Warm absorbing material. The most notable feature in the RGS spectrum is a broad, shallow flux deficit in the energy range $0.65-0.85 \mathrm{keV}$, which cannot be fitted well by an edge. Therefore, we do not confirm the presence of the strong photoelectric edge from O VII of $\tau \sim 0.4$ that Vignali et al. (2004) had reported from the spectral analysis of the previous XMM-Newton observations of Ark 564. It is notable that Matsumoto et al. (2004) find that the OVII edge is very weak $\left(\tau=0.07_{-0.05}^{+0.04}\right)$ in their study of the HETGS spectrum of Ark 564, consistent with our analysis. The study of the UV absorption lines by Crenshaw et al. (2001) also predicts that the O VII edge should be small ( $\tau \sim 0.035$ ), which is again consistent with the results from our analysis.

It is possible that the report of a strong edge in Vignali et al. (2004) is due to their use of a black body model to describe the soft excess in Ark 564, since we also observe a strong absorption feature in the $0.6-0.75 \mathrm{keV}$ band in the best BASELINE+BB model fitting residuals (top panel in Figs. 9 and 10). However, this feature is due to the incorrect form of the continuum model and does not correspond to an intrinsic absorption feature in the spectrum of the source as the RGS data show conclusively.

On the other hand, the broad flux deficit centered at $0.77 \mathrm{keV}$ is similar to the deep troughs that have been detected in the soft $\mathrm{X}$-ray spectra of several Seyfert galaxies and are inferred to be UTA of iron $n=2-3$ absorption lines (e.g. Sako et al. 2001). The location and depth of the UTA implies it is primarily due to material with an ionization parameter of $\xi \sim 10$, and a column density of $N_{\mathrm{Fe}} \sim 10^{16} \mathrm{~cm}^{-2}$ (Behar et al. 2001), corresponding to $N_{\mathrm{H}} \sim 2 \times 10^{20} \mathrm{~cm}^{-2}$ assuming cosmic abundances. The absorption features at the blue end of the UTA region are due to Fe XVI and Fe XVII, and suggest additional material with $\xi \sim 100$, and $N_{\mathrm{H}} \sim 5 \times 10^{20} \mathrm{~cm}^{-2}$. This is broadly in agreement with the estimate of Matsumoto et al. (2004), who also infer an absorber with a range of ionization parameters from their Chandra HETGS spectrum, although our estimate of the ionization parameter of the Fe UTA producing material is larger than theirs.

The absorption feature at $\sim 8 \mathrm{keV}$. We find evidence for an absorption feature in the PN spectrum which can be fitted well by a narrow Gaussian absorption line at $8.14 \pm 0.04 \mathrm{keV}$, with an equivalent width of $\sim 50 \mathrm{eV}$. Absorption lines at energies $>6.5 \mathrm{keV}$ have been detected in the spectra of a few nearby AGN, but with lower equivalent widths (see Bianchi et al. 2005). Recently, Risaliti et al. (2005) reported the detection of a system of Fe XXV and Fe XXVI $\mathrm{K}_{\alpha}$ and $\mathrm{K}_{\beta}$ absorption lines in the spectrum of NGC 1365. Markowitz et al. (2006) also report the detection of a narrow absorption line in the nearby Seyfert IC $4329 \mathrm{a}$ at $7.68 \mathrm{keV}$, which they attribute to Fe XXVI $\mathrm{K}_{\alpha}$ absorption blueshifted to $\sim 0.1 \mathrm{c}$ relative to the systemic velocity. Additionally, absorption features near $\sim 7-8 \mathrm{keV}$, attributed to high-ionization Fe K-shell absorption from material moving at high velocities $(\sim 0.1-0.3 \mathrm{c})$ have been detected in PG and Broad Absorption Line quasars, e.g. Reeves et al. (2003), Chartas et al. (2002), Chartas et al. (2003).

Assuming a narrow line and an energy resolution of the PN detector of $\sim 170 \mathrm{eV}$ at $8 \mathrm{keV}$ (Ehle et al. 2005) we can estimate the significance of this feature from the counting statistics in the PN spectrum to be $\sim 4 \sigma$. However, if the feature we detect is indeed real, then it most probably corresponds to iron absorption from material outflowing with high velocity (see below). In this case, we are in effect fitting the extra absorption line component over many energy bins, where narrow features may occur by chance (i.e. statistical noise). A more conservative estimate of the significance of the line detection can be made if we consider the number of spectral bins over the energy range where one may expect to detect iron absorption lines (Porquet et al. 2004). In our case, we define this energy range to be from $6.7 \mathrm{keV}$ (the Fe XXV K $\alpha$ energy) up to $9.06 \mathrm{keV}$ (the energy of the Fe XXVI K $\alpha$ line from material outflowing at $0.3 \mathrm{c}$ ). There are 81 spectral bins (at 30 counts per bin) in the PN spectrum in this energy range. Following Porquet et al. (2004), we estimate that the probability of detecting the absorption feature at $8.1 \mathrm{keV}$ by chance is only $\sim 2.6 \times 10^{-3}$.

We also carried out a more rigorous test of the significance of the line's detection using Monte Carlo simulations like those performed by Porquet et al. (2004). We used the XSPEC FAKE command to create 1000 synthetic PN spectra corresponding to the PL+NLG best-fitting model, with photon statistics expected from a $100 \mathrm{ks}$ exposure, and grouped to minimum 30 counts per bin. We fitted each synthetic spectrum with a PL+NLG model, and recorded the minimum $\chi_{\mathrm{PL}+\mathrm{NLG}}^{2}$ value. Then we added a narrow absorption line $(\sigma=0.01 \mathrm{keV})$ to the model fit, with the line energy restricted in the range 6.7-9.1 keV. In fact, we stepped the line energy over this energy range, in steps of size $0.1 \mathrm{keV}$, and fitted the model each time to ensure the lowest $\chi_{+ \text {line }}^{2}$ value was found. We then obtained $\Delta \chi^{2}=\chi_{\mathrm{PL}+\mathrm{NLG}}^{2}-\chi_{+ \text {line }}^{2}$ for each synthetic spectrum. Using these 1000 synthetic values we constructed the sample cumulative distribution function of $\Delta \chi^{2}$ in the case of a "blind search" in the $6.7-9.1 \mathrm{keV}$ range, under the assumption there is no real absorption line there. In the case of the Ark 564 PN spectrum, the addition of a narrow absorption line to the PL+NLG model results in a $\Delta \chi^{2}=18.97$. Such a large decrease in $\chi^{2}$ occurred in just 6 of the 1000 synthetic spectra. According to this result, the detection of the absorption line at $\sim 8.1 \mathrm{keV}$ is significant at the $99.4 \%$ confidence level.

We conclude that it is indeed possible that the absorption feature we detect at $8.1 \mathrm{keV}$ is real. This result implies the presence of a second warm absorbing layer, with properties quite different to those mentioned in the previous paragraph. The most 
plausible assumption is that the line corresponds to Fe XXVI $\mathrm{K}_{\alpha}$ at rest energy of $6.97 \mathrm{keV}$. One would also expect to detect the other highly ionized absorption lines at: Fe XXV $\mathrm{K}_{\alpha} \sim 7.64 \mathrm{keV}$, Fe XXV K $\mathrm{K}_{\beta} \sim 8.99 \mathrm{keV}$ and Fe XXVI $\mathrm{K}_{\beta} \sim 9.4 \mathrm{keV}$. There are indeed flux depressions near these energies, but they are not significant and the line associations are marginal. The lack of an observable $\mathrm{Fe} \mathrm{XXV} \mathrm{\textrm {K } _ { \alpha }}$ line implies highly ionized material while the $E W$ of the line we observe implies a high absorbing column of $N_{\mathrm{H}}>10^{23} \mathrm{~cm}^{-2}$ (Risaliti et al. 2005). Under these conditions, the $E W$ of the Fe XXVI K $\mathrm{K}_{\beta}$ line will be 2.5 times smaller than that of Fe XXVI $\mathrm{K}_{\alpha}$, and this can explain the lack of its detection.

However, if the feature we detect is indeed the Fe XXVI $\mathrm{K}_{\alpha}$ line, then it is blue-shifted in the source by $(1+z) \sim 1.17$ (i.e. outflow velocities of $50000 \mathrm{~km} \mathrm{~s}^{-1}=0.17 c$ ). Note that this velocity is not consistent with the recession velocity of Ark 564, which is $\sim 7400 \mathrm{~km} \mathrm{~s}^{-1}$. Consequently, the absorption feature cannot be due to hot Galactic gas, as was recently suggested for some of the absorption signatures in the X-ray spectra of a few AGN (McKernan et al. 2005).

The narrowness of the absorption feature is consistent with the presence of just a single component, i.e. of a single "blob" of material, ejected presumably from the nucleus, as opposed to a continuous flow, which is what one would probably expect, i.e. a stream of gas with a large range of velocities, indicative of its acceleration up to velocities of $\sim 0.1-0.2 \mathrm{c}$. In any case, the absorption feature implies that a very effective acceleration mechanism is in operation in Ark 564. If the bolometric luminosity of the source is indeed comparable to (or even larger than) its Eddington luminosity, perhaps Thomson scattering could provide the necessary acceleration to the warm material.

We finally note that Vaughan et al. (1999) detected an absorption edge of $\tau \sim 0.2$ at $8.6 \mathrm{keV}$ in their combined $A S C A$ and $R X T E$ spectrum of the source. This feature is indicative of the presence of helium-like iron. The authors considered the possibility of absorbing material which lies along the line of sight and found that, in this case, a high ionisation parameter and densities in excess of $10^{23} \mathrm{~cm}^{-2}$ were needed to explain their results. Although the feature we observe in the XMM-Newton spectrum is certainly not an edge, we believe it is interesting that the properties of the absorbing material are similar in both cases.

The $\sim 1 \mathrm{keV}$ emission line. We do not observe any significant emission line features in the $0.9-1.1 \mathrm{keV}$ band, either in the RGS, or in the residuals to the best fitting EPIC model (Figs. 9-11). The addition of such a component to the PN spectral model does not result in a significant improvement in the goodness of fit. We conclude that there is no strong indication of a significant intrinsic emission line at $\sim 0.9-1 \mathrm{keV}$ in the EPIC (and RGS) data of Ark 564. Our results are in agreement with those of Matsumoto et al. (2004) who could not detect a prominent narrow emission line around $1 \mathrm{keV}$ in their Chandra HETGS spectrum. They also ruled out the possibility that the $1 \mathrm{keV}$ feature originates from blends of several narrow emission lines. We believe that, just like with the OVII edge, the previous claims of excess emission at around $1 \mathrm{keV}$ (e.g. Brandt et al. 1994; Turner et al. 1999; Comastri et al. 2001) might have been influenced by the choice of a black body or a power-law model to reproduce the low energy continuum.

The soft excess shape. A solid result of our study is that the soft excess cannot be reproduceded by either a multiple power-law or a power-law plus a single black-body model. In this respect, Ark 564 is different to other NLS1s, like for example Ton S180 (Vaughan et al. 2002) and Mrk 478 (Marshall et al. 2003), which show featureless power-law-like soft excess components. Furthermore, the soft excess emission is probably not resulting from thermal Comptonization either. "Two-temperature" thermal Comptonization models have been used in the past to fit successfully the broad band X-ray spectra of a few NLS1s like e.g. Mrk 896 (Page et al. 2003) and PKS 0558-504 (Brinkmann et al. 2004). This is not the case with Ark 564.

We find that, when we add the 4 Gaussian absorption lines to parametrize the UTA feature, the best description of the soft excess shape in the PN data is provided by two black bodies (with $k T \sim 0.15$ and $\sim 0.07 \mathrm{keV}$ ), or a black-body plus reflection model. The best model fitting residuals in the soft band reduce to the $2 \%$ level, which is comparable to the accuracy with which the instrumental EPIC calibration is currently known. A two black body model fits well the soft excess emission in other NLS1 galaxies as well (e.g. Mrk 359, O’Brien et al. 2001).

However, the temperature of the $k T \sim 0.15 \mathrm{keV}$ black body component is not consistent with that expected from a simple accretion disc. If we assume that the black hole mass in Ark 564 is $2.6 \times 10^{6} M_{\odot}$ (Botte et al. 2004), then a standard accretion disc around it should have a peak temperature $\lesssim 20 \mathrm{eV}$, even if we accept that the system is accreting at its Eddington limit. This is not consistent even with the temperature of the cooler (i.e. the $k T \sim 0.07 \mathrm{keV}$ ) black body component in our model. Clearly the results from the $2 \mathrm{BBs}$ model is at odds with the expectations for a simple accretion disc. This result is in agreement with Gierlinsky \& Done (2004) and Piconcelli et al. (2005), who present a detailed discussion on the problems arising when the soft excess emission in AGN is identified as direct thermal emission from the innermost part of the accretion disc.

A photoionized disc reflection model also fits the overall spectrum well. Our best fit model implies that the iron abundance is consistent with being solar. The measured fraction of reflected over the total observed flux is $\sim 0.45$, close to 0.5 which is expected in the case of gas illuminated by an isotropic source. Interestingly, this model fits well the hard band spectrum as well. Since it reproduces the emission expected from a photoionized accretion disc around a black hole, it includes the iron emission lines at $\sim 6.4-7 \mathrm{keV}$. The fact that the model is consistent with the weak line in the Ark 564 spectrum is mainly due to the fact that it is convolved with a Gaussian in order to simulate the relativistic effects from a disc.

Recently, Crummy et al. (2006) have shown that a relativistically blurred photoionized disc reflection model can fit well the broad band X-ray spectra of a large sample of type 1 AGN. One of the objects in their sample is Ark 564. They also find a good agreement between the model and the $0.3-11 \mathrm{keV}$ spectrum of the source (using data from a past XMM-Newton observation). Their best fit model parameters are somewhat different to ours $(\xi \sim 3100$, iron abundance over solar $\sim 0.5)$. They also detect an absorption edge of $\tau \sim 0.1$ at $\sim 0.68 \mathrm{keV}$. Although this is not as large as the one reported by Vignali et al. (2004), a $\tau \sim 0.1$ edge would still be detected easily in the RGS spectrum. If we take out the black body component from our model, we also observe a strong edge-like feature in the residuals. Since such a strong edge is probably not intrinsic, as we have mentioned before, we believe that the use of a separate black body component, instead of an absorption edge, is a more realistic approach to the modeling of the soft excess in Ark 564. On the other hand, the best fit temperature of the black body component is still quite high $(\sim 0.08 \mathrm{keV})$. Further work, with the use of models more sophisticated than a simple black body, is needed in order to investigate this issue further. 


\section{Conclusions}

We have presented the results from the spectral analysis of a $\sim 100$ ks observation of Ark 564 by XMM-Newton which yielded data with unprecedented quality for this source. Our main aim in this work was the study of the time-average 0.3-11 keV spectrum, using the EPIC PN and MOS data. Using the RGS data, we also investigated the presence of any significant absorption/emission features that could affect the EPIC data. Our results from the detailed study of the RGS spectrum, the timing properties of the source, and from time resolved spectral analysis will be presented in forthcoming papers.

We find that both the PN and MOS spectra above $3 \mathrm{keV}$ are well fitted by a steep power-law of $\Gamma=2.43$.

We observe an iron emission line at $\sim 6.7 \mathrm{keV}$, indicative of the presence of ionized material in the vicinity of the central source. The line is weak $(E W \sim 80 \mathrm{eV})$ and narrow. Although the possibility of a broad line is consistent with the data, it is certainly not required.

At energies below $2 \mathrm{keV}$, both the PN and MOS spectra steepen, and are dominated by a broad soft excess emission. When compared to the hard X-ray power-law, the rise of the soft excess flattens below $\sim 0.6 \mathrm{keV}$.

All our attempts to fit the soft excess were formally not acceptable. However, a model that consists of two black bodies or a black body and a reflection component do fit the soft band EPIC PN spectrum reasonably well, with the amplitude of the remaining model residuals being less than $2 \%$. To a large degree, the fact that these model fits are not statistically acceptable is due to the exceedingly high statistical quality of the data. As a result, at the lowest energies, the model fits are certainly affected by remaining calibration uncertainties. More accurate knowledge of the EPIC PN calibration is needed in order to constrain meaningfully the best fit model parameters in Ark 564.

When compared with the simple accretion disc predictions, the temperature of the black body components in the BASELINE+2BBs model are unreasonably high. For that reason, we believe that the REFL+BB model provides a more physically justified description of the full band X-ray spectrum of Ark 564. Our results suggest the presence of highly ionized material (not surprising, given the high X-ray luminosity of the source) in a geometrically flat disc illuminated by an isotropic source, with solar abundances. The weakness of the iron emission line could be explained by relativistic effects which tend to smooth out the reflected component.

Finally, we do not confirm the presence of the OVII edge and an emission line at $\sim 1 \mathrm{keV}$. Instead, we detect a broad, shallow flux deficit in both the EPIC and RGS spectra which corresponds to the blended unresolved transition arrays of Fe I-XVI, and, for the first time in the case of Ark 564, an absorption line at $\sim 8.1 \mathrm{keV}$ in the PN spectrum. The location and depth of the UTA region implies the presence of warm absorbing material with $N_{\mathrm{H}} \sim(2-5) \times 10^{20} \mathrm{~cm}^{-2}$. If the absorption line corresponds to Fe XXVI K $\alpha$, it suggests the presence of highly ionized, absorbing material of $N_{\mathrm{H}}>10^{23} \mathrm{~cm}^{-2}$, which is moving away from the central source at a high velocity of $\sim 0.17 c$.

Acknowledgements. This work is based on observations with XMM-Newton, an ESA science mission with instruments and contributions directly funded by ESA Member States and the USA (NASA). We gratefully acknowledge travel support through the bilateral Greek-German IKYDA2004 personnel exchange program. P.U. acknowledges support from an EU Marie Curie Fellowship.

\section{References}

Arevalo, P., Papadakis, I. E., Uttley, P., McHardy, I. M., \& Brinkmann, W. 2006, MNRAS, 372, 401

Behar, E., Sako, M., \& Kahn, S. M. 2001, ApJ, 563, 497

Bianchi, S., Matt, G., Nicastro, F., Porquet, D., \& Dubau, J. 2005, MNRAS, 357, 599

Boller, T., Brandt, W. N., \& Fink, H. 1996, A\&A, 305, 53

Botte, V., Ciroi, S., Rafanelli, P., \& Di Mille, F. 2004, AJ, 127, 3168

Brandt, W. N., Fabian, A. C., Nandra, K., Reynolds, C. S., \& Brinkmann, W. 1994, MNRAS, 271, 958

Brinkmann, W., Arevalo, P., Gliozzi, M., \& Ferrero, E. 2004, A\&A, 415, 959

Chartas, G., Brandt, W. N., Gallagher, S. C., \& Garmire, G. 2002, ApJ, 579, 169

Chartas, G., Brandt, W. N., \& Gallagher, S. C. 2003, ApJ, 595, 85

Crenshaw, D. M., Kraemer, S. B., Turner, T. J., et al. 2002, ApJ, 566, 187

Crummy, J., Fabian, A. C., Gallo, L., \& Ross, R. R. 2006, MNRAS, 365, 1067

Comastri, A., Stirpe, G., Vignali, C., et al. 2001, A\&A, 365, 400

Dickey, J. M., \& Lockman, F. J. 1990, ARA\&A, 28, 215

Done, C. 2002, in Roy. Soc. of London Phil. Tr. A, 360, Issue 1798, X-ray astronomy in the new millennium, ed. R. D. Blandford, A. C. Fabian, \& K. Pounds, 1967

Edelson, R., Turner, T. J., Pounds, K., et al. 2002, ApJ, 568, 610

Ehle, M., Breitfellner, M., Gonzales Riestra, R., et al. 2005, XMM-Newton Users Handbook, http://xmm.vilspa.esa.es/xmm_user_support/ documentation/uhb/

Fabian, A. C., Rees, M. J., Stella, L., \& White, N. E. 1989, MNRAS, 238, 729

Gierlinski, M., \& Done, C. 2004, MNRAS, 349, 7L

Gliozzi, M., Brinkmann, W., Räth, C., et al. 2002, A\&A, 391, 875

Grevesse, N., \& Sauval, A. J. 1998, SSRv, 85, 161

Grupe, D. 1996, Ph.D. Thesis, Univ. Göttingen

Haardt, F., \& Maraschi, L. 1993, ApJ, 413, 507

Hasinger, G. 1997, in Imaging and Spectroscopy of Cosmic Hot Plasmas, ed. F. Makino, \& K. Mitsuda (Tokyo: UAP), 263

Lodders, K. 2003, ApJ, 591, 1220

Leighly, K. M. 1999a, ApJS, 125, 297

Leighly, K. M. 1999b, ApJS, 125, 317

Markowitz, A., Reeves, J. N., \& Braito, V. 2006, ApJ, 646, 738

Marshall, H. L., Edelson, R. A., Vaughan, S., et al. 2003, AJ, 125, 459

Matsumoto, C., Leighly, K. M., \& Marshall, H. L. 2004, ApJ, 603, 456

McClintock, J. E., \& Remillard, R. A. 2005, in Compact Stellar X-ray Sources, ed. W. H. Lewin, \& M. van der Klis (Cambridge: Cambridge Univ. Press)

McKernan, B., Yaqoob, T., \& Reynolds, C. S. 2005, MNRAS, 361, 1337

Merloni, A. 2003, MNRAS, 341, 1051

Nayakshin, S., Kazanas, D., \& Kallman, T. R. 2000, ApJ, 537, 833

O’Brien, P. T., Page, K., Reeves, J. N., et al. 2001, MNRAS, 327, L37

Osterbrock, D. E., \& Pogge, R. W. 1985, ApJ, 297, 166

Page, K. L., O'Brien, P. T., Reeves, J. N., \& Breeveld, A. A. 2003, MNRAS, 340,1052

Page, K. L., Turner, M. J. L., Done, C., et al. 2004, MNRAS, 349, 57

Papadakis, I. E., Brinkmann, W., Negoro, H., \& Gliozzi, M. 2002, A\&A, 382, L1

Piconcelli, E., Jimenez-Bailón, E., Guainazzi, M., et al. 2005, A\&A, 432, 15

Porquet, D., Reeves, J. N., Uttley, P., \& Turner, T. J. 2004, A\&A, 427, 101

Pounds, K., Done, C., \& Osborne, J. P. 1995, MNRAS, 277, L5

Pounds, K., Edelson, R., Markowitz, A., \& Vaughan, S. 2001, ApJ, 550, L15

Puchnarewicz, E. M., Mason, K. O., Siemiginowska, A., et al. 2001, ApJ, 550, 644

Reeves, J. N., O’Brien, P. T., \& Ward, M. 2003, ApJ, 593, L65

Risaliti, G., Bianchi, S., Matt, G., et al. 2005, ApJ, 630, L129

Romano, P., Mathur, S., Turner, T. J., et al. 2004, ApJ, 602, 635

Ross, R. R., \& Fabian, A. C. 2005, MNRAS, 358, 211

Sako, M., Kahn, S. M., Behar, E., et al. 2001, A\&A, 365, L168

Stuhlinger, M., et al. 2006, ESA SP-604, 937

Titarchuk, L. 1994, ApJ, 434, 313

Turner, T. J., George, I. M., \& Netzer, H. 1999, ApJ, 526, 52

Turner, T. J., Romano, P., George, I. M., et al. 2001, ApJ, 561, 131

Vaughan, S., \& Fabian, A. C. 2004, MNRAS, 348, 1415

Vaughan, S., Reeves, J., Warwick, R., \& Edelson, R. 1999, MNRAS, 309, 113

Vaughan, S., Boller, T., Fabian, A. C., et al. 2002, MNRAS, 337, 247

Vaughan, S., Edelson, R., Warwick, R. S., \& Uttley, P. 2003, MNRAS, 345, 1271

Vignali, C., Brandt, W. N., Boller, T., Fabian, A. C., \& Vaughan, S. 2004, MNRAS, 347, 854

Wang, T., Brinkmann, W., \& Bergeron, J. 1996, A\&A, 309, 81

Wilms, J., Allen, A., \& McCray, R. A. 2000, ApJ, 542, 914 